\section{MACE RATE FOLLOWING NORMAL REGADENOSON STRESS PERFUSION CARDIOVASCULAR MAGNETIC RESONANCE: A SINGLE CENTRE EXPERIENCE}

${ }^{*}$ G Lewis, J Tan, H Mathias, W Bradlow, R Steeds, B Holloway. University Hospitals Birmingham, UK

10.1136/heartjnl-2016-309668.13

Stress perfusion cardiovascular magnetic resonance (SPCMR) is now established in the investigation of myocardial ischaemia. Various pharmacological options exist for inducing stress conditions. Regadenoson is an A-2A agonist with many advantages over Adenosine, including easier administration and fewer side effects. Whilst the use of Regadenoson has been studied in nuclear myocardial perfusion imaging (MPI), its efficacy has not been as robustly documented in SPCMR. Our centre has a large nuclear MPI service, with a smaller but rapidly growing stress perfusion CMR service. The aim of this study was to determine the major adverse cardiac event (MACE) rate following a normal Regadenoson SPCMR examination.

A retrospective RIS (Radiology Information System) search was performed over a 2 year period $(2013$ - 2014) which yielded 156 SPCMR studies. 95 were excluded due to alternative/undocumented pharmacological stress agents, positive results for inducible ischaemia or failure to complete stress perfusion sequences. This yielded a total cohort of 61 patients who had a normal Regadenoson SPCMR study (this included well documented artefacts such as dark-rim). Basal, mid and apical short axis views were obtained during first pass of contrast (Gadovist, $0.2 \mathrm{mg} / \mathrm{kg}, 6 \mathrm{ml} / \mathrm{second}$ ) at peak stress (400 micrograms Regadenoson). Rest perfusion was repeated $15 \mathrm{~min}$ after the initial contrast injection. All patients were scanned on a Siemens $1.5 \mathrm{~T}$ Avanto MRI scanner. All patients were followed up for at least 1 year for MACE, i.e. cardiovascular death, myocardial infarction or coronary revascularisation.

Out of 61 patients who had a normal Regadenoson SPCMR study, 1 patient experienced MACE within the follow up period (intra-operative death during cardiac transplantation). Excluding this, no patients suffered adverse events during the follow up period. In conclusion, a normal Regadenoson stress perfusion CMR examination is associated with a very low MACE rate within 2 years.

\section{PEAK TURBULENT KINETIC ENERGY ASSESSED BY CARDIAC MAGNETIC RESONANCE CORRELATES BETTER THAN AORTIC VALVE AREA WITH LEFT VENTRICULAR PARAMETERS IN AORTIC STENOSIS}

\begin{abstract}
${ }^{1, *} \mathrm{M}$ Loudon, ${ }^{1} \mathrm{M}$ Bissell, ${ }^{1} \mathrm{~V}$ Stoll, ${ }^{2} \mathrm{P}$ Dyverfeldt, ${ }^{2} \mathrm{C}$ Carlhäll, ${ }^{2} \mathrm{~T}$ Ebbers, ${ }^{1} \mathrm{~A}$ Hess, ${ }^{3}$ B Prendergast, 'S Neubauer, 'S Myerson. 'University of Oxford Centre for Clinical Magnetic Resonance Research, Department of Cardiovascular Medicine, University of Oxford; ${ }^{2}$ Center for Medical Image Science and Visualization (CMIV) and Linköping University, Sweden; ${ }^{3}$ Department of Cardiovascular Medicine, St Guy's and St Thomas' NHS Foundation Trust, London
\end{abstract}

\subsection{6/heartjnl-2016-309668.14}

Introduction Previous studies have shown only modest correlation between aortic valve area (AVA) and left ventricular mass in aortic stenosis (AS), likely due to the variable additional afterload from the aortic and systemic circulation.

Cardiac magnetic resonance (CMR) has developed sequences to measure turbulent kinetic energy (TKE) in the proximal aorta, derived from time resolved three-dimensional (4D) flow imaging. It represents energy dissipation and irreversible pressure loss. Work in the same patient cohort demonstrated peak TKE is higher in bicuspid than tricuspid AS, probably due to larger ascending aortas. We assessed peak systolic TKE in patients with wide ranging AS (mild to severe) and correlated it with left ventricular (LV) parameters.

Methods 22 patients with tricuspid AS (mean age $72.1 \pm 8.6$ years, mean indexed valve area (AVA) $0.55 \pm 0.18 \mathrm{~cm}^{2} / \mathrm{m}^{2}$ ) and 20 with bicuspid AS (age $65.2 \pm 8.5$, indexed AVA $0.77 \pm 0.42$ $\mathrm{cm}^{2} / \mathrm{m}^{2}$ ) were scanned. Peak systolic TKE was measured using specialist analysis software and systolic LV longitudinal strain with proprietary feature-tracking software.

Results LV mass index was the only measure of LV function that correlated with AVA: inverse correlation $(r)=-0.404 ; \mathrm{p} 0.006$ Peak TKE had a stronger correlation with LV mass ( $\mathrm{r} 0.53$; $\mathrm{p}$ 0.001), especially in bicuspid AS ( $\mathrm{r} 0.83$; p 0.0001). Peak TKE also correlated with indexed LV end diastolic volume ( $\mathrm{r} \mathrm{0.486; \textrm {p }}$ 0.003 ), stroke volume ( $\mathrm{r} 0.564 ; \mathrm{p} 0.0004)$ and stroke work ( $\mathrm{r}$ $0.58 ; \mathrm{p} \mathrm{0.0003)}$. This correlation was especially strong in BAV AS (Table 1). No correlation with LV ejection fraction or systolic longitudinal strain was found.

\begin{tabular}{|c|c|c|c|c|c|c|}
\hline \multicolumn{7}{|c|}{ Correlations with TKE } \\
\hline & \multicolumn{2}{|l|}{ AS } & \multicolumn{2}{|l|}{ TAV AS } & \multicolumn{2}{|c|}{ BAV AS } \\
\hline & $r$ & $p$ & $r$ & $p$ & $r$ & $\mathbf{p}$ \\
\hline LV mass index & 0.530 & 0.001 & 0.203 & 0.391 & 0.830 & 0.0001 \\
\hline LVEDVi & 0.486 & 0.003 & -0.126 & 0.5976 & 0.688 & 0.005 \\
\hline SVi & 0.564 & 0.0004 & -.044 & 0.853 & 0.784 & 0.001 \\
\hline LVEF & -0.087 & 0.621 & 0.072 & 0.762 & 0.026 & 0.928 \\
\hline Stroke work index & 0.58 & 0.0003 & 0.21 & 0.373 & 0.833 & 0.0002 \\
\hline
\end{tabular}

Conclusions Peak TKE is a promising tool to better understand the variable effects of AS on the LV, beyond simply severity of AS. The effect is particularly strong in bicuspid AS, who have larger aortas - the likely mechanism for increased TKE.

\section{CARDIAC MAGNETIC RESONANCE IMAGING IN END STAGE RENAL DISEASE USING T1 AND FEATURE- TRACKING}

1,2E Rutherford, ${ }^{1, *} \mathrm{~K}$ Mangion, ${ }^{1} \mathrm{C} \mathrm{McComb},{ }^{1} \mathrm{M}$ Talle, ${ }^{2} \mathrm{AD}$ Struthers, ${ }^{1} \mathrm{C}$ Berry, ${ }^{1} \mathrm{~PB}$ Mark. ${ }^{1}$ BHF Glasgow Cardiovascular Research Centre, Glasgow; ${ }^{2}$ University of Dundee, Division of Cardiovascular \& Diabetes Medicine, Dundee

\subsection{6/heartjnl-2016-309668.15}

Introduction Premature cardiovascular death is the leading cause of mortality amongst End Stage Renal Disease (ESRD) patients. Imaging myocardial fibrosis in ESRD is challenging as gadolinium contrast agent use is contraindicated. Global longitudinal strain (GLS) and native T1 relaxation time may be more informative than ejection fraction (EF) in this patient group.

Methods 33 haemodialysis patients and 28 matched healthy controls underwent CMR at 3T (Magnetom Verio, Siemens, Erlangen, Germany). GLS, strain rate (SR) and early diastolic strain rate (EDSR) were calculated using feature-tracking software (Diogenes Image Arena, Munich, Germany). T1 maps were acquired using an optimised MOLLI investigational prototype 